\title{
Antimicrobial susceptibility testing of escherichia coli isolated from selected dairy farms in and around mekelle, Ethiopia
}

\begin{abstract}
A cross sectional study was conducted from November 2014 to April 2015 in selected dairy farms in and around Mekelle, Ethiopia with the objective of determining the antimicrobial susceptibility pattern of Escherichia coli. From a total of 300 raw milk samples collected, $75(25 \%)$ were found to be positive for Escherichia coli organisms by culture method. In this study, the antimicrobial susceptibility pattern revealed that all the isolates were found to be $100 \%$ susceptible to gentamicin followed by kanamycin (92\%), sulphamethoxazoletrimthoprim $(76 \%)$ on the other hand, in this study, all the isolates were found to be $100 \%$ resistant to penicillin $\mathrm{G}$ followed by amoxicillin (84\%) and tetracycline (60\%). Therefore, in this study gentamicin was the most effective drug against $E$. coli infection followed by kanamycin and sulphamethoxazole-trimethoprim. To safeguard the quality of raw milk, people engaged in milk and dairy production chain should be trained for hygienic practices.
\end{abstract}

Volume 6 Issue I - 2018

\author{
Gebremedhin Yohannes \\ Jigjiga University, Ethiopia
} Correspondence: Gebremedhin Yohannes, DVM, MSc, Jigjiga
University, Jigjiga, Ethiopia, Tel +251914800882 , Email gebyo2005@gmail.com

Received: January 22, 2018 | Published: February 19, 2018

Keywords: antimicrobial susceptibility, escherichia coli, mekelle, milk

Abbreviations: CLSI, clinical and laboratory standards institute; CSA, central statistical agency; CVM, college of veterinary medicine; Lbs, libras; Ml, milliliter

\section{Introduction}

Milk is the fluid normally secreted by female mammals for the nourishment of their young ones. It is a compulsory part of daily diet for the expectant mothers as well as growing children. Milk and milk products are ideal foods for all age groups in both rural and urban people all around the world. ${ }^{1}$ Milk is defined to be the lacteal secretion, practically free from colostrums, obtained by the complete milking of one or more healthy cows, 5 days after and 15 days before parturition. ${ }^{2}$ Raw untreated milk is still used by large number of farm families and workers and by a growing segment of the general population who believe that the milk is not only safe but also imparts beneficial health effects that are destroyed by pasteurization. ${ }^{3}$

Milk is considered a complete and nutritious food; not only for the new-born mammal and for the human beings, but it is considered as a good medium for many microorganisms. ${ }^{3,4}$ It starts its journey in the udder of a mammal as a sterile substance, but as it passes out of the teat, it is inoculated by the animal's normal flora. ${ }^{5}$ Being a nutritionally balanced food stuff with a low microbial load when drawn from the udder of a healthy cow, milk gets contaminated at various stages including the cow itself, the milker's hand or milking equipment, storage vessels and water supply particularly when used for adulteration. ${ }^{6-8}$ Milk being a major constituent of human diet, can serve as a good medium for the growth of many microorganisms especially bacterial pathogens, therefore its quality control is considered essential to the health and welfare of a community. The threat posed by diseases spread through contaminated milk is well known and the epidemiological impact of such diseases is considerable. The presence of these pathogenic microorganisms in milk has emerged as a major public health concern especially for those individuals who still drink raw milk. ${ }^{9}$
Milk is virtually a sterile fluid when secreted into alveoli of udder. However, beyond this stage of production, microbial contamination might generally occur from three main sources; within the udder, exterior to the udder and from the surface of milk handling and storage equipments, but the surrounding air, feed, soil, feces and grass are also possible sources of contamination. Raw milk is usually colonized by a variety of many zoonotic pathogens such as Campylobacter jejuni, Escherichia coli, Salmonella typhimurium, Listeria monocytogenes, Staphylococcus aureus and Yersinia enterocolitica. Therefore, they represent an important source of food borne pathogens. These pathogens in milk have been linked to the environment in the farm, mixing clean milk with mastitis milk and from livestock. ${ }^{10}$

E. coli frequently contaminates food organism and it is a good indicator of fecal pollution. ${ }^{11}$ Presence of E. coli in milk products indicates the presence of enteropathogenic microorganisms, which constitute a public health hazard. E. coli is among many pathogenic microorganisms which can access to milk and some of dairy products which considered a reliable indicator of contamination by manure, soil and contaminated water. ${ }^{12}$

Therefore, the objectives of this study were:

I. To determine the antimicrobial susceptibility pattern of $E$. coli isolates and

II. To select the best antimicrobial drug treatment against $E$. coli infection.

\section{Materials and methods}

\section{Study area}

The study was carried out from November 2014 to April 2015 in and around Mekelle, Tigray, Ethiopia. Mekelle is the capital of Tigray region, located about 783kilometers north of Addis Ababa. Its geographic location is $13032^{\prime} \mathrm{N}$ and $39033^{\circ} \mathrm{E}$, with human population of about 215,546 . It has an average altitude of 2200 meter above sea 
level, with a mean minimum and maximum monthly temperature of $8.7^{\circ} \mathrm{C}$ and $26.8^{\circ} \mathrm{C}$ respectively. The annual average rainfall in Mekelle is $600 \mathrm{~mm} \mathrm{Hg}$ and more than $70 \%$ of its falls between the months July and august. The long dry season extends from October to May. ${ }^{13}$

\section{Study design and sample size}

A cross sectional study was conducted to isolate, identify and determine antimicrobial susceptibility testing of E. coli from five selected dairy farms in and around Mekelle, Tigray, Ethiopia. Farms were selected purposively based on willingness of the farm owners. A total of 300 cows were sampled from five purposively selected dairy farms.

\section{Study animals and sample collection}

The study animals were cross breed lactating dairy cows that were purposively selected from five dairy farms. A total of 300 samples of raw cow's milk were collected from selected dairy farms. The samples were collected in sterilized containers (bottles) and were brought in ice box to the microbiology laboratory of college of veterinary medicine, Mekelle University, immediately to culture the milk samples for isolation and identification of $E$. coli.

\section{Isolation and identification of Escherichia coli}

The milk samples were streaked on the surface of nutrient Agar plates. The plates were incubated in an inverted position at $37 \mathrm{oc}$ for 24hours. After incubation for 24hours, Gram's staining was made by picking a well isolated colonies and prepared a thin smear on a clean glass slide from the culture for differentiation of Gram positive and Gram negative bacteria and, only those Gram negative bacteria were transferred and streaked

Onto the surface of the MacConkey agar plates for differentiation of lactose fermenting and non-lactose fermenting bacteria and, only those lactose fermenting bacteria having a pinkish color colonies were sub-cultured onto the surface of the Eosin Methylene Blue agar (EMB agar) for purification. Biochemical analysis of $E$. coli isolates were performed using catalase test, oxidation-fermentation (O-F) test, indole test, methyl red (MR) test, citrate test and triple sugar iron (TSI) slant agar test.

\section{Antimicrobial susceptibility pattern}

The antimicrobial drugs used were amoxicillin, gentamicin, kanamycin, penicillin G, sulphamethoxazole trimethoprim and tetracycline. The antimicrobial susceptibility testing was done by the agar disc diffusion method as described by Clinical and Laboratory Standards institute (CLSI, 2013). In brief, a 0.5 Mac-Farland standardized suspension of the bacteria was prepared in $0.85 \%$ sterile normal saline solution. A sterile cotton swab was dipped into the standardized suspension of bacteria and then uniformly streaked over the entire surface of the Mueller- Hinton agar. Then, the paper discs impregnated with a fixed concentration of antibiotics were placed on the agar surface and incubated in an inverted position at $37^{\circ} \mathrm{C}$ for 24hours. After incubation for 24hours, clear zones of inhibition were produced by the bacterial growth and diffusion of the antibiotics and these were measured in millimeter using a caliper and interpreted as susceptible, intermediate and resistant. ${ }^{14}$

\section{Data management and analysis}

The data was entered into excel spread sheet and different statistical models were employed to analyze the data collected using Statistical Package for Social Sciences (SPSS) version 15 software. Descriptive statistics was used to describe the frequency and percentage of the results. Chi-square test was used to check the association of potential risk factors with the occurrence of $E$. coli. The association was taken as significant when p-value is less than 0.05 and not significant when $\mathrm{p}$ - value is greater than 0.05 .

\section{Results}

\section{Isolation and Identification of Escherichia coli}

Isolation and identification of E. coli organisms were conducted on raw cow's milk samples using conventional culture and biochemical analysis. From a total of 300 raw cow milk samples collected, 75 (25\%) were found to be positive for E. coli organisms by culture on Eosin Methylene Blue agar medium. Based on biochemical analysis, E. coli isolates were found to be catalase positive, methyl red positive, indole positive and citrate negative. The isolates were also found to be fermentative or facultative anaerobes and having ability to utilize the three sugars (glucose, sucrose and lactose) on oxidation-fermentation test and triple sugar iron slant agar test, respectively.

\section{Potential risk factors}

In this study, the occurrence of $E$. coli was significantly associated with different stage of lactations $\left(\mathrm{x}^{2}=149.677 ; \mathrm{P}=0.000\right)$ and with different parity numbers $\left(\mathrm{x}^{2}=11.594 ; \mathrm{P}=0.009\right)$ (Table 1$)$. Isolation of E. coli has also be significantly associated with age groups $\left(\mathrm{x}^{2}=6.010\right.$; $\mathrm{P}=0.04)$. The highest percentages of $E$. coli isolates were isolated from early stage of lactation $53(84.1 \%)$ and from cows with four and above parity number $43(32.8 \%)$. On the other hand, the present finding revealed that the association between different farms with the occurrence of $E$. coli organisms were not statistically significant $\left(\mathrm{x}^{2}=5.712 ; \mathrm{P}=0.222\right)$ (Table 2) (Figure 1).

Table I Antimicrobial drugs used and their concentrations

\begin{tabular}{ll}
\hline Antimicrobials & Disk concentrations \\
\hline Amoxicillin & 30 microgram \\
Gentamicin & 10 microgram \\
Kanamycin & 30 microgram \\
Penicillin & 10 microgram \\
Sulpha* & 23.75 and I.25microgram \\
Tetracycline & 30 microgram \\
\hline
\end{tabular}

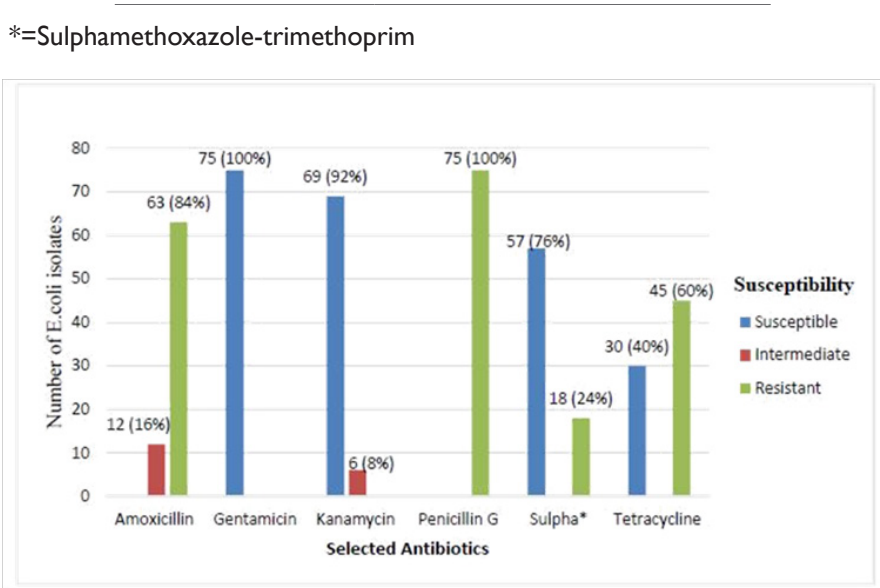

Figure I Antimicrobial susceptibility pattern of Escheriachia coli isolates. 
Table 2 Escherichia coli isolates association with potential risk factors

\begin{tabular}{|c|c|c|c|c|c|}
\hline Risk factors & Positive & Negative & Total & P-value Cl 95\% & \\
\hline \multirow{4}{*}{ Parity } & 1 & $5(13.5 \%)$ & $32(86.5 \%)$ & 37 & \\
\hline & 2 & $8(13.1 \%)$ & $53(86.9 \%)$ & 61 & $X^{2}=11.594$ \\
\hline & 3 & $19(26.8 \%)$ & $52(73.2 \%)$ & 71 & \multirow[t]{2}{*}{$P=0.009$} \\
\hline & $\geq 4$ & $43(32.8 \%)$ & $88(67.2 \%)$ & $|3|$ & \\
\hline \multirow{2}{*}{ Age } & 3-6years & $46(22.1 \%)$ & $162(77.9 \%)$ & 208 & $X^{2}=6.010$ \\
\hline & 7-10years & $29(31.5 \%)$ & $162(77.9 \%)$ & 92 & \multirow[t]{3}{*}{$P=0.04$} \\
\hline \multirow{5}{*}{ Farms } & Kalamino & $30(30 \%)$ & $70(70 \%)$ & 100 & \\
\hline & CVM & $5(14.3 \%)$ & $30(85.7)$ & 35 & \\
\hline & Hidasse & $2(13.3 \%)$ & $13(86.7)$ & 15 & $X^{2}=5.752$ \\
\hline & Kuaha & $10(20 \%)$ & $40(80 \%)$ & 50 & \multirow[t]{2}{*}{$\mathrm{P}=0.222$} \\
\hline & Aga'azi & $28(28 \%)$ & $72(72 \%)$ & 100 & \\
\hline \multirow{4}{*}{ Stage of lactation } & Early & $53(84.1 \%)$ & $10(15.9 \%)$ & 63 & \multirow{3}{*}{$X^{2}=49.677$} \\
\hline & Mid & $15(11.9 \%)$ & III(88.1\%) & 126 & \\
\hline & Late & $7(6.3 \%)$ & $104(93.7 \%)$ & 111 & \\
\hline & Total & $75(25 \%)$ & $225(75 \%)$ & 300 & $P=0.000$ \\
\hline
\end{tabular}

\section{Antimicrobial susceptibility pattern}

A total of $75 \mathrm{E}$. coli isolates were tested against 6 antimicrobials following CLSI guidelines. All E. coli isolates were found to be $100 \%$ susceptible to gentamicin followed by kanamycin $(92 \%)$, sulphamethoxazole-trimthoprim (76\%). On the other hand, all E. coli isolates were found to be $100 \%$ resistant to penicillin $\mathrm{G}$ followed by amoxicillin (84\%) and tetracycline $(60 \%)$. Therefore, gentamicin, kanamycin and sulphamethoxazole-trimethoprim were the most effective drugs against $E$. coli.

\section{Discussion}

Escherichia coli are not only regarded as an indicator of fecal contamination but more likely as an indicator of poor hygiene and sanitary practices during milking and further handling. In this study, a total of 300 raw milk samples were studied and from these 300 raw milk samples, $75(25 \%)$ milk samples were contaminated with E. coli. This is in agreement with the report by Mohanty et al., ${ }^{15}$ who found $(21 \%)$ from India. The current finding was relatively higher as compared to the studies by Lye et al., ${ }^{16}$ and Addo et al., ${ }^{17}$ who reported $8.75 \%$ and $11.2 \%$ from Malaysia and Ghana, respectively. On the other hand, the present study was relatively lower as compared to the studies by Fadaei, ${ }^{18}$ Ali \& Abdelgadir ${ }^{19}$ who reported $69 \%, 63$ $\%$ and $90.67 \%$ from Iran, Khartoum and Tanzania, respectively. This finding difference in the present study from previous studies might be attributed to differences in environmental conditions, management and hygienic practices.

In the present study, the occurrence of $E$. coli has been found significantly associated with different stage of lactations $\left(x^{2}=149.677\right.$; $\mathrm{P}=0.000$ ). The organism was found to be higher in milk taken from cows with early stage of lactation as compared to those in mid and late lactation. The highest percentage of E. coli isolates $(84.1 \%)$ were isolated from early stage of lactation which is relatively higher as compared to the study by Waller et al., ${ }^{20}$ who found $66 \%$ from
Swedish. The occurrence of more E. coli in milk during earlier lactation stage may be due to absence of dry cow therapy and birth related influences in addition to the difference due to management and hygienic practices. The amount of milk ejected is also higher during earlier lactation periods and this cause increased in patency of the teats and decreased local defense factors. ${ }^{21}$

In this study, the occurrence of $E$. coli has also been found significantly associated with parity numbers and different age groups. The highest percentages of $E$. coli isolates were isolated from cows with four or above parity number $(32.8 \%)$ and from cows with age group from seven to ten years $(31.5 \%)$. This could be due to multiple parturition stresses and this ultimately down regulates their immunity, and immunity normally decreases as the animal gets older making more prone to $E$. coli infection. ${ }^{22}$

A total of $75 \mathrm{E}$. coli isolates were tested against 6 antimicrobials based on CLSI guidelines and all $E$. coli isolates were found to be $100 \%$ susceptible to gentamicin followed by kanamycin $(92 \%)$, sulphamethoxazole-trimthoprim $(76 \%)$. On the other hand, all $E$. coli isolates were found to be $100 \%$ resistant to penicillin followed by amoxicillin $(84 \%)$ and tetracycline $(60 \%)$. Relatively similar findings have been reported by Bagre et al., ${ }^{23}$ who found all E. coli isolates were $100 \%$ susceptible to gentamicin, $75 \%$ resistant to amoxicillin and $15 \%$ resistant to sulphamethoxazole-trimethoprim from Burkinafaso. Reuben \& Owuna ${ }^{22}$ were also reported all $E$. coli isolates were $100 \%$ resistant to penicillin and tetracycline, $84.2 \%$ to amoxicillin and sulphamethoxazole-trimethoprim and $89.5 \%$ of the isolates were susceptible to gentamicin from Nigeria. Salehi \& Bonab ${ }^{24}$ were also reported all E. coli isolates were $100 \%$ susceptible to gentamicin, $53 \%$ resistant to amoxicillin, $77 \%$ resistant to kanamycin, $80 \%$ resistant to sulphamethoxazole-trimethoprim and $94 \%$ resistant to tetracycline from Iran. The high level of resistance of penicillin $\mathrm{G}(100 \%)$, amoxicillin (84\%) and tetracycline $(60 \%)$ obtained in this study might be as a result of suboptimal, prolonged 
and interrupted use of antimicrobials for prophylaxis and treatment of infection. Therefore, in this study gentamicin, kanamycin and sulphamethoxazole-trimethoprim were found to be the most effective drugs against $E$. coli infection. ${ }^{24-26}$

\section{Conclusion and recommendations}

The findings obtained in this study revealed that raw cow's milk were found to be highly contaminated with the $E$. coli isolates. Based on the antimicrobial susceptibility pattern, E. coli isolates were found to be highly susceptible to gentamicin, kanamycin and sulphamethoxazole-trimethoprim whereas greatly resistant to penicillin, amoxicillin and tetracycline. Therefore, in this study gentamicin was the most effective drug against $E$. coli infection followed by kanamycin and sulphamethoxazole-trimethoprim.

Based on the above remarks, the following recommendations need to be considered:

I. To ensure the quality of raw milk, everyone engaged in milk and dairy production chain should be trained for hygienic practices.

II. In order to protect consumers from zoonotic diseases, food safety management programmed, the Hazard Analysis and Critical Control point (HACCP), should be implemented and highly considered.

III. Public awareness should be given to the community at risk, whole sellers and distributers.

IV. Regularly teat dips should be applied after milking.

\section{Declaration}

I declare that this research presents the work carried out by myself and does not incorporate without the acknowledgement of any material previously submitted for a degree or diploma in any university; and to the best of my understanding, it does not contain any materials previously published or written by another person except where due reference is made in the text; all substantive contributions by others to the work presented including jointly authored publications, is clearly acknowledged.

\section{Acknowledgements}

I would like to thank the almighty GOD for his fatherly love, being with me and protect me throughout my life.

\section{Conflict of interest}

The author declares no conflict of interest.

\section{References}

1. Javaid SB, Gadahi J, Khaskeli M, et al. Physical and chemical quality of market milk sold at Tandojam, Pakistan. Pakistan Veterinary Journal. 2000;29(1):27-31.

2. Lawley R. Liquid milk products, LFRA Microbiology Handbook of dairy products. 2nd ed. UK: Leather head food Research Association; 2001. p. $1-15$.

3. Lejeune JT, Rajala-Schultz PJ. Food safety: Unpasteurized milk: a continued public health threat. Clinical infection disinfection. 2009;48(1):93-100.

4. Srivastava M. Handbook on milk microbiology. India: Daya Publishing house; 2002. 216 p.
5. Talaro k. Foundations in Microbiology. 5th ed. USA: McGraw-hill Higher education; 2005. p. 815-830.

6. Bonfoh B, Wasem A, Traore A, et al. Microbiological Quality of cow's milk taken at different intervals in Bamako Mali. Food Control. 2003;14(17):495-500.

7. Arora D. Bacteriology of milk. Text book of Microbiology $2^{\text {nd }}$ ed. India: CBS Publishers and Distributors; 2003. $656 \mathrm{p}$

8. Murphy C, Boor K. Trouble shooting sources and causes of high bacteria counts in milk. Dairy food environmental sanitation. 2000:606-611.

9. Foster M. Perennial issues in food safety. In: Cliver DO, editor. Foodborne disease. USA: Academic press; 1990. p. 369-381.

10. Marco M, Well R, Bennik M. Impact of bacterial genomics on determining quality and safety in the dairy production chain. Journal of international dairy production. 2008;18:486-495.

11. Benkerroum N, Bouhal Y, Eiattar A, et al. Occurrence of Shiga toxi producing E. coli $0157: \mathrm{H} 7$ in selected diary and meat products marketed in the city of Rabat, Morocco. Journal of Food Protection. 2004;67(6):1234-1237.

12. Soomro A, Arain M, Khaskheli M, et al. Isolation of Escherichia coli from raw milk and milk products in relation to public health sold under market condition at Tandojam. Pakistan Journal of nutrition. 2002;1(3):151-152.

13. CSA. Central Statstical Agency: Report on monthly average retail prices of goods and services. Stat Bulltn. 2008:416.

14. CLSI. Performance standards for antimicrobial susceptibility testing; twenty-third informational supplement M100-S23. 2013.

15. Mohanty N, Das P, Pany S, et al. Isolation and antibiogram of E. coli isolates from clinical and subclinical cases of bovine mastitis. Veterinary World. 2013;6(10):739-743.

16. Lye YL, Afsah L, Chang S, et al. Risk of Escherichia coli O157:H7 transmission linked to the consumption of raw milk in the state of Selangor, Malaysia. International Food Research Journal. 2013;20(2):1001-1005.

17. Addo K, Mensah G, Aning K, et al. Risk of Escherichia coli O157: H7 transmission linked to the consumption of raw milk in the state of Ghana. International Food Research Journal. 2011;20(2):1001-1005.

18. Fadaei A. Bacteriological quality of raw cow milk in Shahrekord, Iran. Veterinary World. 2014;7(4):240-243.

19. Ali A, Abdelgadir S. Incidence of Escherichia coli in Raw Cow's Milk in Khartoum State. British Journal of dairy Science. 2011;2(1):23-26.

20. Persson Waller K, Bengtsson B, Lindberg A, et al. Incidence of mastitis and bacterial findings at clinical mastitis in Swedish primiparous cows influence of breed and stage of lactation. Vet Microbiol. 2008;134(12):89-94.

21. Thaker C, Brahmbhatt N, Nayak B. Study on occurrence and antibiogram pattern of Escherichia coli from raw milk samples in Anand, Gujarat, India. Veterinary World. 2012;5(9):556-559.

22. Reuben R, Owuna G. In Nasarawa Antimicrobial Resistance Patterns of Escherichia Coli O157: H7 from Nigerian Fermented Milk Samples in State Nigeria. International Journal of Pharmaceutical Science Invention. 2013;2:38-44.

23. Bagré $\mathrm{T}$, Kagambèga $\mathrm{A}$, Bawa $\mathrm{H}$, et al. Antibiotic susceptibility of Escherichia coli and Salmonella strains isolated from raw and curds milk consumed in Ouagadougou and Ziniaré, Burkina Faso. African Joournal of Micribiology Research. 2014;8(10):101-1016.

24. Salehi TZ, Bonab F. Antibiotics Susceptibility Pattern of Escherichia coli Strains Isolated from Chickens with Coli septicemia in Tabriz Province, Iran. International Journal of Poultry Science. 2006;5(7):677-684. 
25. Robert L, Francis S, Athanasia M. Prevalence of Salmonella spp. and Escherichia coli in raw milk value chain in Arusha, Tanzania. American Journal of Research Communication. 2014;2(9):98-105.
26. Rezaei M, Karimi F, Yahyaei M, et al. The survey of microbial total count and prevalence of Escherichia coli in raw milk in Markazi Province, Iran. Animal Veterinary Science. 2013;3(12):474-477. 